\title{
Educación Física en Casa de Calidad. Propuesta de aplicación curricular en Educación Secundaria Obligatoria \\ Quality Physical Education at Home. Curricular implementation proposal in Middle Secondary School \\ ***Rafael Burgueño, ***Alberto Bonet-Medina, ***Álvaro Cerván-Cantón, ***Rubén Espejo, ***Francisco Borja

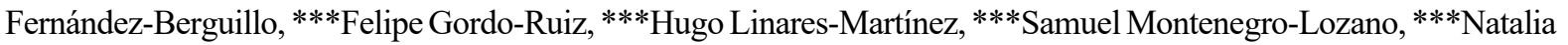 Ordoñez-Tejero, ***Juan José Vergara-Luque, ${ }^{* * *}$ Francisco Javier Gil-Espinosa \\ *Universidad de Almería (España), **Universidad de Granada (España), ***Universidad de Málaga (España)
}

\begin{abstract}
Resumen. Ante situaciones de emergencia que obligan al conjunto de la población - en especial a los adolescentes - a permanecer en casa, la Educación Física (EF) representa una buena estrategia para contribuir a mantener los niveles de actividad física diarios desde casa. Por tanto, el objetivo de este trabajo fue mostrar una propuesta didáctica que, basada en el currículum de Educación Física de educación secundaria obligatoria, contribuya a promocionar la actividad física en casa. Para ello, esta propuesta se fundamenta en un enfoque competencial, incluyendo tanto tecnologías de la información y la comunicación como el establecimiento de retos, con la finalidad de abordar los diferentes contenidos curriculares relacionados con la calidad de vida y salud, condición física y motriz, juegos y deportes, expresión corporal y actividades físicas en el medio natural. La evaluación se plantea mediante una serie de instrumentos (rúbrica, diario, portafolio, hoja de observación y cuestionario) que permitan conocer el grado de consecución de los criterios de evaluación. Después de todo, esta propuesta abre nuevas vías para que el profesorado de EF desarrolle otras propuestas didácticas que faciliten no sólo seguir con las clases de educación física, sino la realización de actividad física en casa.
\end{abstract}

Palabras clave: Actividad física, adolescentes, confinamiento, salud.

Abstract. In emergency situations that force the whole population - particularly adolescents - to stay at home, Physical Education represents an optimal strategy to contribute to adolescents' physical activity at their home. Therefore, this study aims at showing a didactic proposal that, based on Middle Secondary School Physical Education curriculum, promotes adolescents' physical activity at home. For this end, this proposal relies on a competence approach, including both communication and information technologies and the establishment of challenging activities, in order to tackle the different curricular contents related to health and quality of life, physical and motor fitness, games and sports, body expression and physical activities in natural environment. Assessment focuses on a series of instruments (rubric, diary, portfolio, observation sheets, and questionnaire) allowing to evaluate the degree of accomplishment for each assessment criterion. Finally, this proposal offers new avenues for Physical Education teachers to develop other didactic proposals facilitating not only Physical Education classes, but also physical activity at home.

Key words: Physical activity, adolescents, confinement, health.

\section{Introducción}

La actividad física (AF) regular está asociada con una miríada de beneficios para la salud física, psicológica y social para el conjunto de la población y, en especial, para la población adolescente (Guthold et al., 2020). A modo de ejemplo, la AF regular en adolescentes tiene efectos positivos sobre la masa muscular, densidad mineral ósea, colesterol, presión sanguínea (Wilson \& Barnett, 2020), además de prevenir enfermedades tanto cardiovasculares como metabólicas (Alvarez-Pitti et al., 2020). De manera adicional, la AF regular tiene relaciones positivas sobre la vitalidad subjetiva, autoestima, resiliencia, habilidades sociales, contribuyendo, a su vez, a la disminución de niveles de estrés, ansiedad y depresión (Pascoe et al., 2020). No obstante, la investigación ha detectado que la mayoría de los adolescentes no reúnen las recomendaciones de al menos 60 minutos diarios de $\mathrm{AF}$ de moderada a vigorosa y, por tanto, no se benefician de las implicaciones para salud derivadas de su práctica (Guthold et al., 2020). Ante este contexto, la promoción de AF entre la población adolescente se ha convertido en una prioridad de salud a nivel internacional (Organización Mundial de la Salud, 2018).

Fecha recepción: 07-04-20. Fecha de aceptación: 15-06-20 Francisco Javier Gil Espinos javiergil@uma.es
Pese a la elevación del estatus de la AF al de prioridad global de salud, hay un gran número de situaciones (e.g. condiciones meteorológicas adversas, pandemias, etc.) que obligan al conjunto de la población en general y, a la población adolescente en particular, a permanecer en casa (Chen et al., 2020a). En estas situaciones, se hace necesario el mantenimiento de los niveles de AF diaria en casa con la finalidad de mantener un adecuado estado de salud (Chen et al., 2020a). El confinamiento podría aumentar el nivel de inactividad física de los adolescentes (Márquez-Arabia, 2020; MeraMamían et al., 2020). En este sentido, el profesorado de Educación Física (EF) podría desempeñar un rol esencial a la hora de promover entre los adolescentes la AF regular en sus casas, en la medida que el currículum español de EF para educación secundaria obligatoria establece la consolidación de hábitos de cuidado de la salud y, la reducción de conductas que la dañan (e.g. actividades sedentarias, consumo de drogas, alcohol y tabaco) junto con la promoción de AF para la ocupación activa del tiempo libre y de ocio (Real Decreto 1105/2014).

Por tanto, el objetivo de este estudio fue realizar una propuesta didáctica basada en la EF para que la población adolescente practique AF en casa y, en consecuencia, mantenga los niveles de AF diaria. En esta misma línea, esta propuesta pretender representar un punto de referencia a partir del cual pueda ser adaptada a las circunstancias específicas de cada uno, tales como la duración del confinamiento en casa, el nivel tanto de autonomía como de AF del 
alumnado, así como el grado de implicación familiar. Por esta serie de razones, la propuesta didáctica se ha diseñado de manera abierta y flexible, abarcando los tres cursos que actualmente configuran el primer ciclo de educación secundaria obligatoria (i.e. primero, segundo y tercero) de acuerdo con lo establecido por el Real Decreto 1105/2014. Por ello, esta propuesta debe ser entendida como un modelo de referencia para programar la asignatura de EF en Casa, de forma que el trabajo solicitado por el profesorado esté justificado curricularmente, evitando el riesgo de atender propuestas desestructuradas u orientadas exclusivamente a la condición física.

\section{Método}

El presente estudio queda enmarcado dentro de la investigación educativa de tipo documental, lo que sugirió la necesidad de usar un muestro aleatorio simple.

Utilizando como referencia el objetivo planteado en el trabajo, se ha realizado una revisión de la legislación educativa española y la literatura científica en relación con la importancia de la actividad física en casa para adolescentes. Por otro lado, se analizaron diferentes propuestas de actividad física que puedan ser aplicadas en casa y contextualizadas en el currículo oficial.

No se emplearon parámetros temporales como criterios de exclusión de artículos, ni tampoco hubo restricciones en el criterio de idiomas, aceptando sólo artículos derivados de fuentes primarias (i.e. artículos científicos de carácter tanto original como revisiones sistemáticas, propuestas didácticas y ensayos). En este sentido, se realizó una búsqueda en las bases de datos ISI-Web of Science y Scopus dado su carácter multidisciplinar. El logaritmo de búsqueda fue establecido sobre la base de diferentes combinaciones entre los términos: physical activity, exercise, program, adolescents, secondary school, physical education, home, COVID-19y el uso de los booleanos de búsqueda AND, *, OR. No obstante, se debe subrayar que el presente trabajo no pretende ser una revisión exhaustiva de la investigación sobre la educación física en casa, sino un análisis de la importancia que tanto el legislador como la literatura científica otorgan a ambos. Igualmente, se aporta una propuesta genérica de aplicación práctica con el objetivo de que el profesorado pueda adaptarla y concretar. Por ello, los datos fueron identificados, categorizados y analizados de acuerdo con la técnica de análisis de contenido, apoyándose en el paradigma cualitativo de análisis.

\section{Resultados}

\section{Análisis contextual}

A la hora de analizar el contexto de la casa, se debe tener en consideración la gran heterogeneidad presente en relación no sólo con las características específicas de los domicilios, sino también con los diferentes tipos tanto de materiales como de recursos que podrían encontrarse en cada uno de ellos (Del Espino-Hidalgo, 2017). En este aspecto, para la programación y desarrollo de AF en casa, se debe considerar una serie de factores dependientes estrechamente vinculados con el alumnado: a) domicilio, espacio disponible para la realización de $\mathrm{AF}$; b) recursos y materiales; c) apoyo de la familia hacia la $\mathrm{AF}$; d) recomendaciones de $\mathrm{AF}$ en casa.

En cuanto al domicilio, debido a la gran variedad entre sus características, se debe analizar de forma general las distintas posibilidades espaciales que toda vivienda podría ofrecer y, de acuerdo con esta disponibilidad, realizar una propuesta adaptada de AF en casa. En concreto, cualquier vivienda ofrece la posibilidad de, al menos, tener una habitación disponible con espacio aprovechable para la práctica de AF, además de otros tipos de espacios, a priori, aprovechables tales como escaleras, patios o terrazas, aunque sería posible que no todos estos se encontrasen en todas las viviendas. Por otra parte, también habría que considerar las distintas superficies existentes como tipos más o menos deslizantes o más o menos rugosos de solería.

En lo referente a la tipología de recursos y materiales, habría que destacar aquellos recursos y materiales de carácter tecnológico tales como el ordenador, tabletas electrónicas, móviles, televisores o videoconsolas la mayoría de ellos con conexión a internet. De manera adicional, se debe tener en cuenta materiales y recursos de índole más convencional presentes en toda casa, como podría ser toallas, sillas, garrafas de agua, palos de fregona, mesas entre los más destacables.

En relación con el apoyo de la familia hacia la AF en casa, la familia se ha considerado como un factor determinante a la hora de promover la participación en AF de sus miembros menores de edad (Cueto-Martín et al., 2018; Lahti et al., 2020). En concreto, el apoyo mostrado por el padre/madre hacia la AF ha sido percibido por los niños (alumnado) como una fuente de motivación intrínseca, favoreciendo, su participación regular en diferentes AF y, consecuentemente, el desarrollo tanto su competencia motriz como su condición física (Rhodes et al., 2018, 2019). En esta misma línea, se piensa incluso que la práctica de AF compartida y simultánea entre niños y padres/madres podría ser una adecuada estrategia para iniciar y consolidar la AF dentro de la unidad familiar (Cueto-Martín et al., 2018). Para ello, se hace recomendable que la tipología de AF requiera de la participación de, al menos, dos miembros de la familia, además de partir de los intereses comunes de los diferentes miembros que componen la unidad familiar.

Finalmente, la literatura especializada en AF (Chen et al., 2020a, 2020b; de Oliveira-Neto et al., 2020; Hammami et al., 2020; Jiménez-Pavón et al., 2020; Rodríguez et al., 2020; Shirvani \& Rostamkhani, 2020) propone una serie de pautas, consejos y recomendaciones a la hora de practicar AF en casa: a) la AF regular como medida de mantenimiento del estado general de salud, del peso y, como medio para optimizar la respuesta del sistema inmune; b) AF de moderada a vigorosa, evitando la realización de sesiones extenuantes y de larga duración; c) si hay síntomas de fiebre, cualquier tipo de AF debe desaconsejarse; d) la posibilidad de realizar dos sesiones de entrenamiento, siempre y cuando el intervalo de tiempo entre ambas sea superior a tres horas; e) el entrenamiento cardiovascular no deberá ser superior a 60 minutos con una frecuencia máxima cardíaca igual o inferior al $80 \%$ de la máxima; f) evitar tanto el trabajo con cargas máximas como las repeticiones hasta el fallo y agotamiento muscular; g) el mantenimiento de una adecuada hidratación antes, durante 
y después del entrenamiento; h) uso de una variedad de ejercicios seguros, simples y de fácil implementación.

\section{Análisis curricular}

De acuerdo con el Ministerio de Educación y Cultura (1992), una unidad didáctica se conceptualiza como:

Unidad de programación y actuación docente configurada por un conjunto de actividades que se desarrollan en un tiempo determinado, para la consecución de unos objetivos didácticos. Una unidad didáctica da respuesta a todas las cuestiones curriculares al qué enseñar (objetivos y contenidos), cuándo enseñar (secuencia ordenada de actividades y contenidos), cómo enseñar (actividades, organización del espacio y del tiempo, materiales y recursos didácticos) y a la evaluación (criterios e instrumentos para la evaluación), todo ello en un tiempo claramente delimitado

En este sentido, el término integrador hace referencia al ensamblaje de un modo eficaz de los elementos que conforman una unidad didáctica integrada (UDI) con la finalidad de lograr la adquisición de las competencias clave (Clavijo, 2015). De acuerdo con este planteamiento, a la hora de diseñar toda UDI, se deben seleccionar aquellas competencias clave que se pretendan lograr. Tales competencias clave se describen en la Orden ECD/65/2015. No obstante, en la medida que las competencias clave no son directamente medibles, se necesitan unos objetivos didácticos que permitan definir los resultados. En este mismo sentido y, en línea con la Orden $\mathrm{ECD} / 65 / 2015$, esta función es asumida por los criterios de evaluación, los cuales sirven de guía para valorar lo que un estudiante sabe hacer en cada materia y, con ello, el logro de las competencias clave.

En consonancia con el artículo 5 de la citada Orden ECD/ $65 / 2015$, los criterios de evaluación se desglosan en estándares de aprendizaje evaluables, siendo éstos los que permiten valorar el grado de desempeño alcanzado en cada una de las competencias. Por tanto y según Clavijo (2015), en el diseño de toda UDI, se debe tener en cuenta la planificación desde atrás, seleccionando los objetivos didácticos cuya función queda asumida por los indicadores de logro, es decir, por los estándares de aprendizaje evaluables. Estos estándares de aprendizajes evaluables se encuentran operativizados en el Real Decreto 1105/2014. Sin embargo, a la hora de proponer los bloques de contenidos a trabajar desde el área de EF, el artículo 6 bis de la Ley Orgánica para la Mejora de la Calidad Educativa (2013) delega la competencia a las administraciones autonómicas (Méndez-Alonso et al., 2017). Aunque bien es cierto que, dentro de la legislación actual y tras el análisis del Real Decreto 1105/2014, se puede hacer una clara distinción entre cinco bloques de contenidos: a) salud y calidad de vida; b) condición física y motriz; c) juegos y deportes; e) expresión corporal y d) AF en el medio natural.

\section{Mapa curricular}

En consonancia con el análisis curricular expuesto, se va a proceder al diseño del mapa de desempeño curricular, amparado en el Real Decreto 1105/2014, Orden ECD/65/2015, y la Ley Orgánica de Educación (2006) con las modificaciones de la Ley Orgánica para la Mejora de la Calidad Educativa
(2013). Más concretamente, éste va a quedar organizado en tres grandes bloques: a) concreción curricular; b) transposición didáctica y, c) valoración de lo aprendido (Clavijo, 2015).

\section{Concreción curricular}

En la Tabla 1 y Tabla 2, se desarrolla la concreción curricular de la propuesta, indicando los criterios de evaluación, objetivos generales de etapa, objetivos didácticos o de área de $\mathrm{EF}$, contenidos, elementos transversales, competencias clave, interdisciplinariedad, transposición didáctica y evaluación.

Con mayor grado de concreción y de acuerdo con el Real Decreto 1105/2014, esta propuesta didáctica pretende cubrir la consecución de seis objetivos generales de etapa (artículo 11) destacando especialmente aquellos relacionados con el incorporar la EF y la práctica deportiva para favorecer el desarrollo personal y social, así como, el cuidado de la salud personal, social y ambiental (k), el adquirir una preparación básica para el uso responsable de las tecnologías, especialmente las de la información y la comunicación (e), el desarrollar tanto la iniciativa personal como la capacidad de aprender a aprender junto con la toma de decisiones y la confianza en sí mismo (g), el apreciar la expresión artística a través de sus diferentes medios de expresión y representación (1) y, el asumir responsablemente el ejercicio de los derechos y responsabilidades de un ciudadano que participa libremente en un sociedad plural y plenamente democrática (a).

A partir de los objetivos generales de etapa, la presente propuesta plantea, en base al Real Decreto 1105/2014, seis objetivos de área tales como el mejorar el nivel de ejecución y aplicaciones técnicas, teniendo en cuenta el grado de esfuerzo, auto-exigencia y superación (1.4), el utilizar técnicas corporales creativamente considerando la estructura espacio-temporal y la intensidad (2.1), el crear y proponer una secuencia de ritmos corporales ajustado a un ritmo (2.2), el mejorar el nivel de condición física relacionado con la salud con la puesta activa en práctica de métodos básicos para su desarrollo (5.1), así como, el conocer las posibilidades del entorno a la hora de practicar AF y deporte (8.1) y, el uso responsable de las tecnologías de la comunicación y la información (10.1). En esta misma línea, los contenidos curriculares propuestos cubrieron la totalidad de los cinco bloques de contenidos (i.e. salud y calidad de vida, condición física y motriz, juegos y deportes, expresión corporal y AF en el medio natural) descritos por la Orden de 14 de julio de 2016 (Boletín Oficial de la Junta de Andalucía, 2016). De igual manera, esta propuesta didáctica contribuye a las competencias clave de aprender a aprender, en el sentido que permite el desarrollo y el manteniendo de la salud y condición física en casa a través del movimiento; de sentido a la iniciativa y espíritu emprender, en el medida que persigue la autonomía para la realización de las distintas tareas buscando la motivación intrínseca; de matemáticas, ciencias y tecnología, mediante el análisis y conocimiento del propio cuerpo; competencia digital a partir del uso responsable de aplicaciones para la creación y comunicación de diferentes recursos; social y cívica, al crear y compartir retos físicos con sus compañeros/as y, competencia lingüística por medio de la utilización del cuerpo y el lenguaje como medio de expresión de sentimientos, emociones e ideas. 
Tabla 1

Mapa de desempeño curricular relativo a la unidad didáctica

\begin{tabular}{|c|c|c|c|c|}
\hline Estándar de aprendizaje evaluable & Criterios de evaluación & Objetivo de etapa & Contenido & Competencias clave \\
\hline $\begin{array}{l}\text { 1.4. Mejora su nivel en la ejecución y aplicación de las } \\
\text { acciones técnicas respecto a su nivel de partida, } \\
\text { mostrando actitudes de esfuerzo, autoexigencia y } \\
\text { superación. }\end{array}$ & $\begin{array}{l}\text { 1. Resolver situaciones motrices individuales aplicando los } \\
\text { fundamentos técnicos y habilidades específicas, de las } \\
\text { actividades fisico-deportivas propuestas, en condiciones reales o } \\
\text { adaptadas. }\end{array}$ & $\mathrm{k}, \mathrm{g}, \mathrm{a}$ & Juegos y deportes. & CAA, SIEP \\
\hline $\begin{array}{l}\text { 2.1. Utiliza técnicas corporales, de forma creativa, } \\
\text { combinando espacio, tiempo e intensidad } \\
\text { 2.2. Crea y pone en práctica una secuencia de } \\
\text { movimientos corporales ajustados a un ritmo prefijado. }\end{array}$ & $\begin{array}{l}\text { 2. Interpretar y producir acciones motrices con finalidades } \\
\text { artístico-expresivas, utilizando técnicas de expresión corporal y } \\
\text { otros recursos. }\end{array}$ & $1, \mathrm{k}, \mathrm{g}$ & Expresión corporal & CEC, CAA, CSYC \\
\hline $\begin{array}{l}\text { 5.1. Participa activamente en la mejora de sus } \\
\text { capacidades fisicas básicas desde un enfoque saludable, } \\
\text { utilizando los métodos básicos para su desarrollo. }\end{array}$ & $\begin{array}{l}\text { 5. Desarrollar las capacidades físicas de acuerdo con las } \\
\text { posibilidades personales y dentro de los márgenes de la salud, } \\
\text { mostrando una actitud de autoexigencia en su esfuerzo. }\end{array}$ & $\mathrm{k}, \mathrm{a}, \mathrm{g}, \mathrm{e}$ & $\begin{array}{l}\text { Salud y calidad de vida, Condición fisica y } \\
\text { motriz }\end{array}$ & CAA, CMCT \\
\hline $\begin{array}{l}\text { 8.1. Conoce las posibilidades que ofrece el entorno para } \\
\text { la realización de actividades físico-deportivas. }\end{array}$ & $\begin{array}{l}\text { 8. Reconocer las posibilidades que ofrecen las actividades } \\
\text { fisico-deportivas como formas de ocio activo y de utilización } \\
\text { responsable del entorno. }\end{array}$ & $\mathrm{e}, \mathrm{g}, \mathrm{k}$ & $\begin{array}{l}\text { Salud y calidad de vida, Condición física y } \\
\text { motriz, Juegos y deportes, Expresión corporal, } \\
\text { Actividades fisicas en el entorno (casa) }\end{array}$ & CMCT, CAA, CSC. \\
\hline $\begin{array}{l}\text { 10.1. Utiliza las tecnologías de la información y la } \\
\text { comunicación para elaborar documentos digitales } \\
\text { propios (texto, presentación, imagen, video, sonido...), } \\
\text { como resultado del proceso de búsqueda, análisis y } \\
\text { selección de información relevante. }\end{array}$ & $\begin{array}{l}\text { 10. Utilizar las Tecnologías de la Información y la } \\
\text { Comunicación en el proceso de aprendizaje, para buscar, } \\
\text { analizar y seleccionar información relevante, elaborando } \\
\text { documentos propios, y haciendo exposiciones y } \\
\text { argumentaciones de los mismos }\end{array}$ & $\mathrm{a}, \mathrm{e}, \mathrm{g}, \mathrm{k}$ & $\begin{array}{l}\text { Salud y calidad de vida, Condición fisica y } \\
\text { motriz, expresión corporal, juegos y deportes. }\end{array}$ & CD, SIEP \\
\hline
\end{tabular}

Por otra parte, la presente propuesta didáctica también trata de trabajar en pos de la igualdad efectiva entre hombres y mujeres, la salud, la expresión oral y el uso responsable de las TIC y las TAC como elementos trasversales del currículum (Real Decreto 1105/2014). De la misma forma, que se trabajará de forma disciplinar con el área de lengua castellana y literatura, ciencias naturales, música y educación plástica y visual.

En cuanto a la evaluación, la Tabla 2 muestra, en línea con el Real Decreto 1105/2014, anexo I, los diferentes estándares de aprendizaje considerados y su peso en el proceso de evaluación, junto con su respectivo criterio de evaluación.

\section{Modelo Unidad Didáctica}

La Tabla 2 muestra el modelo de UDI relativo al primer ciclo de la Educación Secundaria Obligatoria para el que está dirigido esta propuesta didáctica. Así pues, las situaciones de emergencia (e.g. pandemia) suponen una oportunidad para llevar la EF a las casas del alumnado y poner en práctica todos los recursos TIC que faciliten el contacto con el alumnado y la evaluación de la propia UDI. En esta unidad didáctica, se plantean actividades para abordar cada uno de los cinco bloques de contenidos. Por tanto, se trabaja la condición motriz, condición física-salud, la expresión corporal, los juegos y deportes, y el medio natural a través de actividades que pueden ser realizables por el alumnado en

Tabla 2 .

Propuesta esquematizada de unidad didáctica integrada de educación física para el primer ciclo de educación secundaria obligatoria

Justificación: La necesidad de llevar la educación física a las casas y a los hogares de nuestro alumnado da origen a esta unidad. La pandemia no sé supera sentados y esperando a queCiclo: $1^{\circ}$ Ciclo ESO pase, debemos conseguir que el alumnado se mueva e intentar que sus familias lo hagan con ellos. Es el momento de conocer cómo realizar ejercicio físico desde casa.

(a) 26 de Diciember Competencias clave: Siguiendo el artículo 2 del Real Decreto 1105/2014, de 26 de diciembre, las competencias los objetivos de etapa que vamos a trabajar en esta unidad son: a, e, g, k, l. $\quad$ de etapa que vamos a trabajar en esta unidad son: CAA, CSC, CEC, SIEP, CMCT, CD, CL.

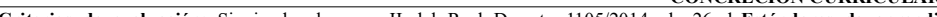

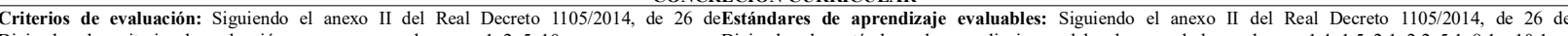

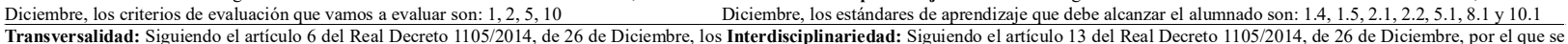
Transversalidad: Siguiendo el artículo 6 del Real Decreto 1105/2014, de 26 de Diciembre, los Interdisciplinariedad: Siguiendo el artículo 13 del Real Decreto 1105/2014, de 26 de Diciembre, por el que se
elementos transversales de etapa que vamos a trabajar en esta unidad son: 2, 3 y $4 \quad$ establece las asignaturas de la ESO, en esta unidad vamos a trabajar de forma interdisciplinar: LCL, MAT, CN,

Atención a la diversidad: Siguiendo el artículo 9 del Real Decreto 1105/2014, de 26 de Diciembre, el cual establece medidas y programas para la atención a la diversidad, esta unidad va a permitir que el alumnado reciba un aprendizaje lo más individualizado posible, ya que el espacio en el cual se desarrolla la unidad es ideal para este tipo de alumnado.

Contenidos (bloques). Según la Orden de 14 de Julio de 2016 (Boletín Oficial de la Junta de Andalucía, 2016), se trabajarán los siguientes contenidos del currículo:

1. Salud y calidad de vida: Alimentación saludable, fomento de actitudes de vida sana y activa, fomento y práctica de fundamentos de higiene postural, uso responsable de las tecnologías de la información. 2 . Condición física y motriz: acondicionamiento fisico general a través del desarrollo de las cualidades fisicas relacionadas con la salud (aerobic, circuitos de y apps). 3. Juegos y Deportes: Fundamentos técnico y habilidades motrices específicas de las actividades físico-deportivas individuales y colectivas y juegos alternativos 4. Expresión corporal: Utilización de técnicas de expresión corporal de forma creativa combinando espacio, tiempo e intensidad; juegos rítmicos, malabares. 5. Actividades físicas en el medio natural: Técnicas básicas de orientación, interpretación de la simbología, identificación mapaterreno/terreno-mapa, orientación del mapa, juegos de pistas y orientación.

TRANSPOSICIÓN DIDACTICA

Sesiones/Actividades:

$1^{\circ}$ Sesión: Retos de pilates o yoga, rutinas de estiramientos y conciencia postural

$2^{\circ}$ Sesión: Rutinas autocargas, juegos de condición física y retos de condición fisic

$3^{\circ}$ Sesión: Malabares, retos con papel WC y suavibol

$4^{\circ}$ Sesión: Retos en Tik Tok, retos de baile en Just Dance, coreografías baile-fitness y teatro de sombras.

$5^{\circ}$ Sesión: Circuito orientacion en casa, aprender a medir distancias en pasos y talleres teóricos-prácticos deportes en el medio natural.

Metodología: Tomando como referencia un enfoque competencial (ver Granero-Gallegos, 2020), se utilizará una metodología activa inductiva donde el alumno tiene un papel activo en el proceso de enseñanza-aprendizaje. Se fomentará la resolución de problemas tanto mediante la búsqueda como a través del aprendizaje ensayo-error. El objetivo fundamental de la metodología será que el alumnado sea el protagonista de su aprendizaje, para lo que es fundamental hacer un buen uso de las diferentes técnicas y estilos de enseñanza.

Escenarios didácticos: Casa

Recursos: App brújula, metro, papel, boli, dispositivo móvil, app Tik-Tok, app Just Dance, app Youtube, lámpara o flexo, calcetines, pared, pelotas de tenis o de arroz, rollo de papel higiénico, bote de suavizante o botella, esterilla o toalla, mochila con peso, cámara, botellas de agua, sillas y TRX casero

\begin{tabular}{|c|c|c|c|c|c|}
\hline \multicolumn{6}{|c|}{ VALORACIÓN DE LO APRENDIDO } \\
\hline $\begin{array}{c}\text { Estándares de } \\
\text { aprendizaje (\%) }\end{array}$ & Instrumento & Nivel 4 (9-10) & Nivel 3 (7-8) & Nivel 2 (5-6) & Nivel 1 (0-4) \\
\hline $\begin{array}{l}\mathbf{2 . 1}(15 \%) \\
\mathbf{2 . 2}(15 \%)\end{array}$ & Rúbrica de evaluación & $\begin{array}{c}\text { Utiliza técnicas corporales, de forma } \\
\text { creativa, combinando espacio, tiempo e } \\
\text { intensidad. Además, crea y pone en práctica } \\
\text { una secuencia de movimiento ajustada a un } \\
\text { ritmo. }\end{array}$ & $\begin{array}{l}\text { Utiliza técnicas corporales, de forma creativa, } \\
\text { combinando espacio, tiempo e intensidad. } \\
\text { Además, crea y pone en práctica una secuencia de } \\
\text { movimiento, pero no está ajustada a un tiempo. }\end{array}$ & $\begin{array}{l}\text { Utiliza técnicas corporales, de forma } \\
\text { creativa, combinando espacio, tiempo } \\
\text { e intensidad, pero no crea ni pone en } \\
\text { práctica una secuencia de movimiento. }\end{array}$ & $\begin{array}{l}\text { No utiliza técnicas corporales, } \\
\text { de forma creativa combinando } \\
\text { espacio, tiempo e intensidad. }\end{array}$ \\
\hline $\begin{array}{l}\mathbf{1 . 4}(15 \%) \\
\mathbf{5 . 1}(15 \%)\end{array}$ & Diario de & $\begin{array}{c}\text { Participa en la mejora de las capacidades } \\
\text { fisicas básicas utilizando los métodos básicos } \\
\text { para su desarrollo, mejorando su nivel de } \\
\text { partida y mostrando actitudes de esfuerzo y } \\
\text { superación. } \\
\end{array}$ & $\begin{array}{l}\text { Participa en la mejora de las capacidades físicas } \\
\text { básicas utilizando los métodos básicos para su } \\
\text { desarrollo, mejorando su nivel de partida, pero } \\
\text { muestra una mala actitud. }\end{array}$ & $\begin{array}{c}\text { Participa en la mejora de las } \\
\text { capacidades fisicas básicas utilizando } \\
\text { los métodos básicos para su } \\
\text { desarrollo, pero sin mejorar su nivel } \\
\text { de partida. }\end{array}$ & $\begin{array}{l}\text { No participa en la mejora de } \\
\text { las capacidades fisicas básicas. }\end{array}$ \\
\hline $\begin{array}{l}\mathbf{1 . 5}(15 \%) \\
\mathbf{8 . 1}(15 \%)\end{array}$ & Cuestionario & $\begin{array}{c}\text { Conoce las posibilidades que presenta el } \\
\text { entorno para la realización de actividades } \\
\text { fisico-deportivas. Además, pone en práctica } \\
\text { técnicas básicas de orientación y de creación } \\
\text { de mapas. }\end{array}$ & $\begin{array}{l}\text { Conoce las posibilidades que presenta el entorno } \\
\text { para la realización de actividades físico- } \\
\text { deportivas, y pone en práctica técnicas de } \\
\text { orientación, pero no crea mapas. }\end{array}$ & $\begin{array}{c}\text { Conoce las posibilidades que presenta } \\
\text { el entorno para la realización de } \\
\text { actividades físico-deportivas, pero no } \\
\text { pone en práctica técnicas de } \\
\text { orientación. }\end{array}$ & $\begin{array}{l}\text { No conoce las posibilidades } \\
\text { que presenta el entorno para la } \\
\text { realización de actividades } \\
\text { fisico-deportivas. }\end{array}$ \\
\hline $\mathbf{1 0 . 1}(10 \%)$ & Hoja de observación & $\begin{array}{l}\text { Utiliza las tecnologías de la información y la } \\
\text { comunicación para elaborar y compartir } \\
\text { documentos digitales después de realizar } \\
\text { resultado del proceso de búsqueda, análisis y } \\
\text { selección de información. }\end{array}$ & $\begin{array}{l}\text { Utiliza las tecnologías de la información y la } \\
\text { comunicación para elaborar y compartir } \\
\text { documentos digitales, pero no hay proceso de } \\
\text { búsqueda, análisis y selección de información. }\end{array}$ & $\begin{array}{l}\text { Utiliza las tecnologías de la } \\
\text { información y la comunicación para } \\
\text { elaborar documentos digitales, pero no } \\
\text { los comparte. }\end{array}$ & $\begin{array}{l}\text { No utiliza las tecnologías de la } \\
\text { información y la comunicación } \\
\text { para elaborar documentos } \\
\text { digitales }\end{array}$ \\
\hline
\end{tabular}


Tabla 3.

\begin{tabular}{|c|c|c|}
\hline Bloque de contenidos & Ejemplo propuestas didácticas & Materiales y recursos \\
\hline $\begin{array}{l}\text { B1: Salud y calidad de } \\
\text { vida }\end{array}$ & $\begin{array}{l}\text { - Retos de pilates o yoga. } \\
\text { - Rutinas estiramientos. } \\
\text { - Conciencia postura corporal } \\
\text { cotidiana. }\end{array}$ & $\begin{array}{l}\text { - Esterilla o toalla. } \\
\text { - Cámara de fotos }\end{array}$ \\
\hline $\begin{array}{l}\text { B2: Condición física y } \\
\text { motriz }\end{array}$ & $\begin{array}{l}\text { - Rutinas autocargas. } \\
\text { - Rutinas con material de casa. } \\
\text { - Juegos de condición fisica } \\
\text { - Retos de condición física }\end{array}$ & $\begin{array}{l}\text { - Esterilla o toalla } \\
\text { - Mochila con peso } \\
\text { - Botellas de agua } \\
\text { - Sillas } \\
\text { - TRX casero }\end{array}$ \\
\hline B3: Juegos y deportes & $\begin{array}{l}\text { - Malabares } \\
\text { - Retos con papel WC. } \\
\text { - Suavibol }\end{array}$ & $\begin{array}{l}\text {-Pelotas de tenis, de arroz o } \\
\text { calcetines } \\
\text { - Rollo de papel higiénico } \\
\text { - Bote de suavizante o botella y } \\
\text { pelota }\end{array}$ \\
\hline $\begin{array}{l}\text { B4: Expresión } \\
\text { corporal }\end{array}$ & $\begin{array}{l}\text { - Retos en Tik Tok } \\
\text { - Retos de baile en Just Dance } \\
\text { - Coreografias baile-fitness } \\
\text { - Teatro de sombras }\end{array}$ & $\begin{array}{l}\text { Dispositivo móvil } \\
\text { App Tik-Tok } \\
\text { App Just Dance } \\
\text { App Youtube } \\
\text { Lámpara o flexo y pared. }\end{array}$ \\
\hline $\begin{array}{l}\text { B5: Actividad fisica } \\
\text { en el medio natural }\end{array}$ & $\begin{array}{l}\text { - Circuito orientación en casa } \\
\text { - Aprender a medir distancias en } \\
\text { pasos } \\
\text { - Talleres teóricos-prácticos deportes } \\
\text { en el medio natural }\end{array}$ & $\begin{array}{l}\text { App brújula } \\
\text { Metro } \\
\text { Papel y boli }\end{array}$ \\
\hline
\end{tabular}

casa, teniendo en cuenta los recursos de los que disponen. La transposición didáctica se enmarcó dentro de un enfoque competencial (ver Granero-Gallegos, 2020) por medio de la propuesta de una yincana de bienvenida (i.e. tarea competencial) que se prepara mediante la realización de las diversas $\mathrm{AF}$ a desarrollar en casa por el alumnado. La evaluación del alumnado se plantea a través de diferentes instrumentos, como son la rúbrica, el diario o portafolio, la hoja de observación y el cuestionario. Este conjunto de instrumentos de evaluación será utilizado para verificar que el alumnado ha conseguido alcanzar los criterios de evaluación marcados. El propósito fundamental es aportar al alumnado recursos que le permitan realizar actividad física en casa y en familia, buscando alcanzar un estilo de vida saludable.

\section{Propuesta práctica a desarrollar}

El área de educación física puede ser una de las que más dificultades ocasione a la hora de enviar trabajos para casa de forma telemática, sin embargo, es recomendable solicitar tareas que impliquen el movimiento, utilizando únicamente recursos materiales caseros y recursos digitales. Se debe evitar el uso de materiales o propuestas que tengan algún riesgo de accidente o lesión. Sería recomendable hacer indicación al respecto al alumnado y familias. El profesorado, conocedor de la realidad de su alumnado y familias, debería aportar unas normas básicas de seguridad en la realización de actividad física en casa y, proponer alternativas. La propuesta que se presenta

Tabla 4 con las manos. $\underline{\mathrm{rcBwIQ14}}$

B5: Actividad física en el Circuito orientación en casa: de mapa. a continuación pretende aportar una visión globalizada de la diversidad de actividades que se pueden proponer para realizar durante el confinamiento, evitando la supremacía de actividades exclusivamente orientadas a la condición física.

En este sentido, las diferentes tareas, que integran la presente propuesta didáctica, han sido diseñadas sobre la base de actividades que supongan un reto y desafío físico para el alumnado (ver Tabla 3). En este sentido, la decisión de establecer diversos retos físicos se tomó sobre la base de que éstos favorecen el desarrollo tanto la motivación al logro del estudiante como sus destrezas relacionadas con la competencia motriz (Hastie et al., 2019), mejorando su responsabilidad, tiempo de práctica y esfuerzo percibido (Hortigüela-Alcalá et al., 2018), además de estimular su interés hacia la tarea y fomentar la diversión y el disfrute, así como, evitar el aburrimiento en las clases de EF (Dismore \& Bailey, 2011). En concreto, la Tabla 4 presenta una serie de retos planteados por bloques de contenidos, además de establecer una relación práctica y motivadora entre las tareas y la situación de confinamiento.

En el bloque uno, se persigue la mejora y reforzamiento de la postura corporal, tan difícil de mantener en esta situación, con la finalidad de mejorar la flexibilidad mediante la actividad de Yoga. En el bloque dos, se pretende que el alumno esté en movimiento por medio de retos motivantes que hagan conseguir la mejora del nivel de condición física mediante ejercicios físicos variados y de fácil realización en casa. En el bloque tres, se plantean retos con papel higiénico que fomentan la práctica deportiva de diversos deportes con

Propuesta de actividades a desarrollar por cada bloque de contenido B1: Salud y calidad de Reto Yoga: Realizar una serie o rutina de posturas de yoga, aumentando cada $\mathrm{App}$ vida vez más la dificultad de estas, hasta conseguir un nivel más avanzado. Cartas de posturas Yoga https://yogakiddy.com/juego-yoga-gratis-

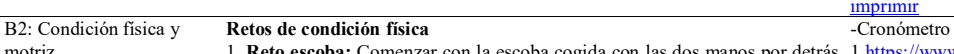

motriz 1 . Reto escoba: Comenzar con la escoba cogida con las dos manos por detrás 1. https://www.youtube.com/watch?v=de la espalda y acabar en la misma posición pasándola por delante. 9TYAPV0kO4 2. Reto burpees: Series de 1 minuto, se realiza 1 burpee y se descansa hasta
completar el minuto, en cada serie se aumenta en uno el número de burpees a $5 . h$ htps://www.youtube.com/watch?v=41N realizar. Termina cuando el alumno no consigue realizar todos los burpees en 6 bKO-NVI realizar. Termina cuando el alumno no consigue realizar todos los burpees en $\frac{6 \mathrm{bKO}-\mathrm{NVI}}{\mathrm{https}: / / \mathrm{ww} \text {.youtube.com/watch?v=bql6sI }}$
el tiempo. 3. Reto sentadilla isométrica: consiste en aguantar el mayor tiempo posible $\underline{\underline{U} 2 \mathrm{~A} 7 \mathrm{k} \& \mathrm{t}=2 \mathrm{~s}}$ en posición de sentadilla isométrica.

4. Reto plancha isométrica: consiste en aguantar el mayor tiempo posible en posición de plancha isométrica.

5. Reto Bring sally up: consiste en seguir el ritmo de la canción realizando un 5. Reto Bring sally up: consiste en seguir el ritmo de la canción realizando un
fondo de brazos o una sentadilla, subiendo cuando la canción marca UP y \begin{tabular}{llllll}
\multicolumn{1}{c}{ bajando cuando marca DOWN, manteniendo la posición entre transiciones. } \\
\hline B3: Juegos y deportes & Retos rollo de papel de WC: (todo con papel de WC) Rollo de papel WC
\end{tabular} 1. Realizar 10 toques con las piernas. $\quad$ Papelera

2. Encestar en una papelera sin mirar desde una distancia de 10 pasos.

3. Malabares (se pueden utilizar calcetines, según tamaño corporal). Realizar el "8" " "cascada". Practicar progresión con vídeos explicativos.

4. Soltar el rollo desde arriba por detrás de la cabeza y cogerlo con las manos por debajo de las piernas.

5. Rollo puesto en el suelo, y tienes que levantarlo con los pies para cogerlo

6. Desde posición erguida, inclinamos el tronco hacia delante, de forma que cadera y espalda quedan en ángulo recto. El rollo lo colocamos en las

\begin{tabular}{lll} 
& lumbares, y hay que darle la vuelta sin usar las manos. & \\
\hline B4: Expresión corporal & Retos en Tik Tok: vídeos con retos que deben aprender e intentar repetirlos. & App tiktok \\
& 1. Reto Baile con los pies & App Youtube
\end{tabular} 1. Reto Baile con los pies

https://www.youtube.com/watch?v=xHBwE7h8plE

-Variante para 3: https://www.youtube.com/watch?v=3WGPVPUb2Y

2. Reto de los gestos: https://www.youtube.com/watch?v=11nn6c4leok

3. Reto de baile con las manos: https://www.youtube.com/watch?v=Sv-

4. Creación de reto propio y compartir con los compañeros.

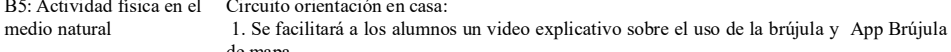

2. Se les pedirá que diseñen un mapa de su casa.

3. Tendrán que responder a unas preguntas mediante el uso de la brújula

(adjuntando foto) en referencia a la situación de los lugares de casa. Recurso video explicativo:

(Por ejemplo: Indica cuántas ventanas hay en la zona Sur de casa. Indica la https://www.youtube.com/watch?v=KM0

orientación en la que se encuentra la puerta de casa.)

4. Un miembro de la casa tendrán que esconder 3 objetos e indicarlos con una

cruz en el mapa. El niño deberá de encontrar estos objetos escondidos.

App Youtube $\underline{\text { PBQCYJIA }}$ 
material alternativo y, a la vez, se trabajan habilidades motrices de diferentes juegos. En el bloque cuatro, se presenta el uso de la aplicación Tik Tok para abordar el trabajo tanto de la expresión corporal como de la propia creatividad. En el último y quinto bloque, se propone la puesta en práctica de un circuito de orientación en casa, empleando la brújula como uso de las TIC, así como, la creación de un mapa de la vivienda para poder desarrollar la práctica.

\section{Propuestas genéricas para la $\mathrm{AF}$ en casa}

La Tabla 5 recoge una serie de propuestas de carácter genérico en relación con la práctica de AF en casa. Así pues, éstas se caracterizan por su facilitad a la hora de ser adaptadas, implementadas y contextualizadas por el profesorado de EF, dependiendo de las peculiaridades específicas de su alumnado y de los recursos materiales a su disposición. En particular, se destacan desafíos como el reto AF en casa 30 días o el reto \#saltazapatos para trabajar la salud y la calidad de vida, así como actividades que combinan los desplazamientos con el golpeo a un globo-pelota o la rayuela practicada en familia como medios para abordar los contenidos vinculados con la condición física y motriz. De forma adicional, se propone la práctica tanto de spikeball modificado como del ringo y de los bolos para desarrollar contenidos asociados con juegos y deportes. Además, se hace una propuesta de dramatizaciones y representaciones de películas

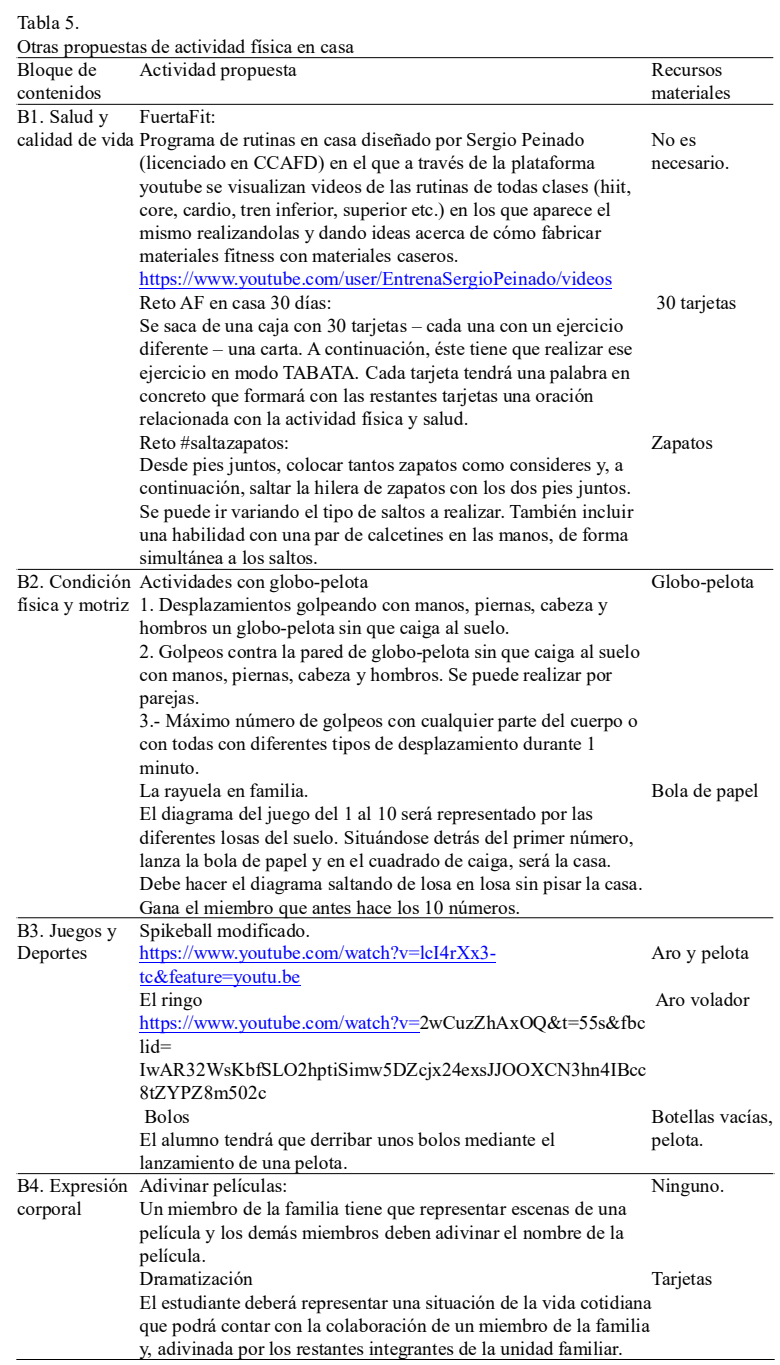

que involucran a la unidad familiar como vehículo a la hora de tratar los contenidos referidos a la expresión corporal. Se utilizan como tecnologías para el aprendizaje y el conocimiento las herramientas tik-tok, just dance, youtube y app de brújula.

\section{Conclusiones}

Este trabajo pretende proporcionar una propuesta didáctica fundamentada en el currículum de EF para que el alumnado pueda realizar AF en casa y, al mismo tiempo, seguir de alguna manera con las clases de EF. Esta propuesta destaca tanto por su carácter innovador por el uso adecuado de las nuevas tecnologías de la información y de la comunicación como por su enfoque competencial y empleo de retos, así como por su fácil implementación por parte del alumnado en su domicilio. De modo adicional, esta idea abre nuevas vías para que otros profesionales de la enseñanza de la EF desarrollen otras propuestas didácticas que faciliten no sólo seguir con las clases de EF, sino la realización de AF con intensidad moderada en casa cuando las condiciones obligan al alumnado a permanecer confinados en casa.

\section{Futuras líneas de investigación}

La situación de excepcionalidad y confinamiento que estamos viviendo puede ser aprovechada como una oportunidad para avanzar hacia la integración de las tecnologías de la información y comunicación como recurso educativo que permite mejorar los niveles de actividad física del alumnado, así como su motivación y tratamiento individualizado, avanzando hacia el logro de una EF de Calidad como establece la Organización de las Naciones Unidad para la Educación, la Ciencia y la Cultura (UNESCO, 2015). Podríamos indicar que sería necesario avanzar hacia una Educación Física en Casa (EFC) de Calidad. Por tanto, sería conveniente un análisis e investigación en relación con la AF y su tipología realizada por el alumnado durante este período, al objeto de tener la posibilidad de avanzar en la mejora y, porqué no, aprovechamiento de ese conocimiento incluso para tiempos donde el confinamiento no sea obligatorio.

\section{Referencias}

Alvarez-Pitti, J., Casajús-Mallén, J.A., Leis-Trabazo, R., Lucía,A., López de Lara, D., Moreno-Aznar, L. A., \& RodríguezMartínez, G. (2020). Exercise as medicine in chronic diseases during childhood and adolescence. Anales de Pediatría, 92,4-11. doi:10.1016/j.anpedi.2020.01.010

Chen, P., Mao, L., Nassis, G P., Harmer, P., Ainsworth, B. E., \& Li, F. (2020a). Wuhan coronavirus (2019-nCoV): The need to maintain regular physical activity while taking precautions. Journal of Sport and Health Science, 9(2), 103-104. doi:10.1016/j.jshs.2020.02.001

Chen, P., Mao, L., Nassis, G P., Harmer, P., Ainsworth, B., \& Li, F. (2020b). Returning Chinese school-aged children and adolescents to physical activity in the wake of COVID-19: Actions and precautions. Journal of Sport and Health Science. doi:10.1016/j.jshs.2020.04.003

Clavijo, J. M. (2015). Las competencias clave como elemento 
integrador y esencial del currículo. Consejería de Educación. Junta de Andalucía.

Cueto-Martín, M. B., DeLa Cruz, J.C., Morales-Ortiz, E., \& PérezDíaz, C. (2018). Effect of joint physical activity on the physical condition of parents and children. Journal of Human Sport and Exercise, 13(2), 415-429. doi:10.14198/jhse.2018.132.12

de Oliveira-Neto, L., Elsangedy, H. M., Tavares, V.D. O., Behm, D. G., \& da Silva-Grigoletto, M. E. (2020). \#TrainingInHometraining at home during the COVID-19 (SARS-COV2) pandemic: Physical exercise and behavior-based approach. Brazilian Journal of Exercise Physiology, 12(3), 23-31.

Del Espino-Hidalgo, B. (2017). Mid-sized cities in the centre of Andalusia. An approach to an urban-territorial phenomeno. Revista de Estudios Regionales, 7585(108), 165-191.

Dismore, H., \& Bailey, R. (2011). Fun and enjoyment in physical education: Young people's attitudes. Research Papers in Education,26(4),499-516. doi:10.1080/02671522.2010.484866

Granero-Gallegos,A. (2020). Hacia la enseñanza competencial, una propuesta desde la Educación Física. Editorial Universidad de Almería.

Guthold, R., Stevens, GA., Riley,L. M., \& Bull,F.C.(2020). Global trends in insufficient physical activity among adolescents: A pooled analysis of 298 population-based surveys with 1.6 million participants. The Lancet Child and Adolescent Health,4(1), 23-35. doi:10.1016/S2352-4642(19)30323-2

Hammami,A.,Harrabi,B.,Mohr,M., \& Krustrup,P.(2020). Physical activity and coronavirus disease 2019(COVID-19): Specific recommendations for home-based physical training. Managing Sport and Leisure. doi:10.1080/ 23750472.2020.1757494

Hastie, P. A., Rudisill, M. E., Boyd, K., \& Johnson, J. L. (2019). Examining the pathway to motor skill sompetence in a mastery motivational climate: An appreciative inquiry. Research Quarterly for Exercise and Sport, 90(3), 259-269. doi:10.1080/ 02701367.2019.1593921

Hortigüela-Alcalá, D., Salicetti, A., \& Hernández, J. (2018). Motivational contrast in Physical Education depending on caloric expenditure. Revista Internacional de Medicina y Ciencias de la Actividad Fisica y del Deporte, 18(72), 621635. doi:10.15366/rimcafd2018.72.002

Jiménez-Pavón, D., Carbonell-Baeza, A., \& Lavie, C. J. (2020). Physical exercise as therapy to fight against the mental and physical consequences of COVID-19 quarantine: Special focus in older people. Progress in Cardiovascular Diseases. doi:10.1016/j.pcad.2020.03.009

Lahti,A., Rosengren, B. E., Nilsson, J. Å., Peterson, T., \& Karlsson, M. K. (2020). Age-, gender- and family-related factors were the most important socio-ecological associations with physical activity in children with a mean age of eight years. Acta Paediatrica, International Journal of Paediatrics, 109(4), 853-854. doi:10.1111/apa.14776

Ley Orgánica 2/2006, de 3 de mayo, deEducación. Boletín Oficial de Estado (2006).

Ley Orgánica 8/2013, de 9 de diciembre, para la Mejora de la CalidadEducativa. Boletín Oficial del Estado 27548 (2013).

Márquez-Arabia, J. J. (2020). Inactividad física, ejercicio y pandemia COVID-19. Viref Revista de Educación Física, $9(2), 63-71$.

Méndez-Alonso, D., Pérez-Pueyo, Á., Méndez-Giménez, A., Fernández-Río, J., \& Prieto-Saborit, J. (2017). Análisis del desarrollo curricular de la educación física en la enseñanza secundaria obligatoria: Comparación de los currículos autonómicos. Retos: Nuevas Tendencias En Educación Física, Deportey Recreación, 2041(31), 82-87.

Mera-Mamían,A. Y., Tabares-Gonzalez, E., Montoya-Gonzalez, S., Muñoz-Rodriguez, D. I., \& Monsalve Vélez, F. (2020). Recomendaciones prácticas para evitar el desacondicionamiento físico durante el confinamiento por pandemia asociada a COVID-19. Universidady Salud,22(2), 166-177.https://doi.org/10.22267/rus.202202.188

Ministerio de Educación y Cultura (1992). Orientaciones didácticas. Secundaria obligatoria. Cajas Rojas.

Real Decreto 1105/2014, por el que se establece el currículo básico de la Educación Secundaria Obligatoria y del Bachillerato. Boletín Oficial delEstado(2014).

Orden de 14 de julio de 2016, por la que se desarrolla el currículo correspondiente a la Educación Secundaria Obligatoria en la Comunidad Autónoma de Andalucía. Boletín Oficial de la Junta deAndalucía (2016).

Orden ECD/65/2015, de 21 de enero, por la que se describen las relaciones entre las competencias, los contenidos y los criterios de evaluación de la educación primaria, la educación secundaria obligatoria y el bachillerato. Boletín Oficial de Estado(2015).

Organización de las Naciones Unidad para la Educación, la Ciencia. y la Cultura. (2015). Educación física de calidad(EFC): Guía para los responsables políticos. Ediciones UNESCO.

Organización Mundial de la Salud. (2018). Global action plan on physical activity 2018-2030. More active people for a healthier world.

Pascoe, M., Bailey,A. P., Craike, M., Carter, T., Patten, R., Stepto, N., \& Parker,A. (2020). Physical activity and exercise in youth mental health promotion: Ascoping review. BMJOpen Sport andExercise Medicine, 6(1), 1-11. doi:10.1136/bmjsem-2019000677

Rhodes, R. E., Beauchamp, M. R., Blanchard, C. M., Bredin, S. S., Warburton, D. E., \& Maddison, R. (2018). Use of in-home stationary cycling equipment among parents in a familybased randomized trial intervention. Journal of Science and Medicine in Sport, 21(10), 1050-1056. doi:10.1016/ j.jsams.2018.03.013

Rhodes, R. E., Blanchard, C. M., Quinlan, A., Naylor, P. J., \& Warburton, D.E. R. (2019). Family physical activity planning and child physical activity outcomes: A randomized trial. American Journal of Preventive Medicine, 57(2), 135-144. doi:10.1016/j.amepre.2019.03.007

Rodríguez, M. Á., Crespo, I., \& Olmedillas, H. (2020). Exercising in times of COVID-19: What do experts recommend doing within four walls? Revista Española de Cardiología. doi:10.1016/j.rec.2020.04.001

Shirvani, H., \& Rostamkhani, F. (2020). Exercise considerations during coronavirus disease 2019(COVID-19) Outbreak: A narrative review. Journal of Military Medicine, 22(2), 161168./doi:10.30491/JMM.22.2.161

Wilson, B., \& Barnett,L. M.(2020). Physical activity interventions to improve the health of children and adolescents in out of home care-A systematic review of the literature. Children and Youth Services Review, 110. doi:10.1016/ j.childyouth.2020.104765 